effective model for inducing nausea without vomiting can be used to study effects of nausea on GI function together with correlations with objective physiological and brain activity markers. The markers identified may help to reduce and refine animal experiments.

Competing interests None declared.

\section{PWE-052 PHENOTYPING THE EARLY MORNING RUSH (EMR) IN PATIENTS WITH DIARRHOEA PREDOMINANT IRRITABLE BOWEL SYNDROME (IBS-D)}

doi:10.1136/gutjnl-2012-302514d.52

${ }^{1} \mathrm{~K}$ Garsed, ${ }^{*}{ }^{2} \mathrm{M}$ Hastings, ${ }^{1} \mathrm{C}$ Lam, ${ }^{2} \mathrm{P}$ Whorwell, ${ }^{1} \mathrm{R}$ Spiller. ${ }^{1}$ Nottingham Digestive Diseases Centre NHIR BRU, University of Nottingham, Nottingham, UK; ${ }^{2}$ Neurogastroenterology and Motility, Wythenshawe Hospital, Manchester, UK

Introduction A proportion of patients with IBS-D describe frequent bowel movements first thing in the morning. While most clinicians recognise the phenomenon there is currently no definition of this "early morning rush" and no data describing the subgroup of patients who experience this disabling symptom which frequently impacts on their quality of life and ability to work

Methods 119 patients meeting the Rome 3 criteria for IBS-D and 20 age and sex matched healthy volunteers (HV) completed a 1-week stool diary, hospital anxiety and depression score (HADS), and personal health questionnaire (PHQ-12). They also completed an IBS severity score (IBSSS) and IBS quality of life score (IBSOOL) as part of entry into a clinical trial. Whole gut transit (WGT) was measured during the placebo arm using the radio-opaque marker method. EMR was defined as $\geq 2$ bowel movements within $1 \mathrm{~h}$, between midnight and midday. Patients and HV were divided into 3 subgroups, those with $\leq 2$ days/week $E M R=$ normal morning rush (NMR), those with 3-4 days/week EMR = moderate morning rush (MMR), and those with 5-7 days/week with EMR = severe morning rush (SMR). Results $16 \%$ of patients had MMR and $15 \%$ of patients had SMR, all HV had NMR. Those with SMR had greater psychological distress (sum of the HADS score) than those with NMR, 19.11 $( \pm 1.8)$ vs $13.48( \pm 0.8) \mathrm{p}=0.021$, and more somatic symptoms (PHO-12), $9.2( \pm 0.9)$ vs $6.5( \pm 0.4) \mathrm{p}=0.02$. The SMR group had greater mean daily stool frequency than those with NMR and MMR $5.6(3.5-8.2)$ vs $2.14(1.7-3.0)$ and $3.7(2.7-4.4) \mathrm{p}<0.0001$ with a higher IBSSS $366( \pm 18.4)$ vs $296.6( \pm 9.4)$ and 295.9 ( \pm 17.2$)$ $\mathrm{p}=0.005$, this was accompanied by a lower IBSQOL $334.3( \pm 21.1)$ vs 467.3 ( \pm 17.9$)$ and $467.3( \pm 33.4) \mathrm{p}=0.004$. WGT (in hours) was significantly faster in the SMR group $7(5.5-15)$ vs $19(10-39)$ in the NMR and $16(8.5-24.5)$ in the MMR groups $p=0.03$.

Conclusion Around $1 / 3$ of IBS patients suffer from early morning rush. Those severely affected represent a group with a worse quality of life, greater psychological distress and more somatic symptoms. This is associated with faster whole gut transit and increased stool frequency. A third of the patients with SMR dropped out of the trial compared to $<10 \%$ in the other groups making this a challenging subgroup to study.

Competing interests None declared.

\section{PWE-053 CYCLIC VOMITING SYNDROME IN 28 PATIENTS: DEMOGRAPHICS, FEATURES AND OUTCOMES}

\section{doi:10.1136/gutjn-2012-302514d.53}

${ }^{1} \mathrm{~L}$ Lee, * ${ }^{1} \mathrm{~L}$ Abbott, ${ }^{2} \mathrm{~J}$ Burnett, ${ }^{3} \mathrm{~S}$ Anderson, ${ }^{1} \mathrm{~S}$ Moodie. ${ }^{1}$ Department of Gastroenterology, Epsom and St Helier University Hospitals NHS Trust, London, UK; ${ }^{2}$ Guy's and St Thomas' NHS Foundation Trust, London, UK; ${ }^{3}$ Department of Gastroenterology, Guy's and St Thomas' NHS Foundation Trust, London, UK

Introduction Cyclic Vomiting syndrome (CVS) is a condition characterised by recurrent, stereotyped attacks of intense nausea and intractable vomiting with no identifiable organic cause. The diagnosis of CVS has been helped by the Rome III diagnostic criteria, however, the condition is still a very heterogeneous group of symptoms and there have been very little studies analysing in detail patient-reported features. There is now some evidence for the role of tricyclic antidepressants in managing these patients, but as yet, there is no evidence on the long-term outcomes of patients treated conservatively.

Methods This was a retrospective cohort study of all patients treated at two London teaching hospitals. Information was gathered by means of inpatient case notes, clinic letters and telephone interviews with standardised questions.

Results 28 patients were identified, 17 with adult onset-CVS and 11 with childhood-onset CVS. Overall, $54.5 \%$ of patients noted that each attack occurred at the same time of day, usually between 4:00-6:00. In the adult-onset CVS cohort, the average age of onset was 30.9 years; the prevalence of headache was $57.1 \%$. Vomiting attacks occurred on average 10 times a year with a mean duration of $55.3 \mathrm{~h}$. In the childhood-onset CVS cohort, the average age of onset was 5.7 years and the prevalence of headaches was $44.4 \%$. Vomiting attacks occurred on average 25.5 times a year with a mean duration of $54.5 \mathrm{~h}$. During acute admissions patients rated morphine-based medications and ondansetron as most effective. Long-term, $65.4 \%$ of our patients were treated conservatively and of these $42.1 \%$ felt their symptoms had improved and a further $23.8 \%$ noted that their symptoms had resolved completely.

Conclusion Cyclic Vomiting Syndrome is a debilitating illness with a high impact on patient's quality of life. There is a high association with anxiety/depression as well as headaches. Morphine based medications are the most efficacious symptomatic acute treatment. Conservative management has a role to play in the long-term treatment of these patients. The prognosis of these patients is good with nearly $70 \%$ of patients having an improvement in their symptoms and nearly a quarter having resolution of the condition after 7 years.

\section{Abstract PWE-053 Table 1}

\begin{tabular}{lccc}
\hline & Overall & Adults & Children \\
\hline No/year, mean (SD) & $16.3(22.9)$ & $10.0(12.1)$ & $25.5(31.4)$ \\
Duration, mean hours (SD) & $55(43.8)$ & $55.3(39.9)$ & $54.5(50.7)$ \\
Vomiting freq, mean (SD) & $4.1(2.6)$ & $3.5(1.7)$ & $4.6(3.3)$ \\
Duration of recovery phase, mean hours (SD) & $53.2(47)$ & $59.1(43.6)$ & $45(53.2)$ \\
\hline
\end{tabular}

Competing interests None declared.

\section{PWE-054 THE MANAGEMENT OF CYCLIC VOMITING SYNDROME: A SYSTEMATIC REVIEW OF 1141 CASES}

doi:10.1136/gutjnl-2012-302514d.54

L Lee, ${ }^{*}$ L Abbott, B Mahlangu, S Moodie. Epsom and St Helier University Hospitals NHS Trust, London, UK

Introduction Cyclic Vomiting syndrome (CVS) is characterised by recurrent attacks of intractable vomiting with no identifiable organic cause. It can take years before CVS is diagnosed, furthermore there have only been a handful of case studies looking at management strategies to reduce the duration/frequency or intensity of cyclic vomiting attacks. The objectives of this review were to identify associated clinical features that would help suggest a diagnosis of CVS and to review the literature to identify management strategies with the highest efficacy.

Methods A literature search was performed using the databases MEDLINE via Ovid (1948 to October 2011) and EMBASE (1980 to October 2011). The search terms included "Cyclic" and "Vomiting". 
Data from adult-onset to paediatric-onset CVS were included. Data were extracted into a standardised form.

Results The systematic search yielded 33 papers with 1141 cases of cyclic vomiting syndrome. All but one paper were retrospective studies. Both adult-onset and paediatric-onset CVS had a high association with headaches/migraines and psychiatric conditions such as anxiety/depression. Furthermore, in children travel sickness was noted in $28.3 \%$. The mean duration and frequency of attacks were higher in adult-onset CVS compared to paediatric-CVS. Overall remission was achieved in $73.2 \%$ of cases. When tricyclic antidepressants were used, $75.5 \%$ of adult-onset CVS patients had a response $(n=237)$ and $67.6 \%$ of paediatric onset-CVS patients $(\mathrm{n}=244)$. In adult-onset CVS, 37 patients had been treated with sumatriptan with a response rate of $56.8 \%$. In paediatric-onset CVS, 91 patients had been treated with propranolol and amitriptyline resulting in a response rate of $86.8 \%$. There were no studies focusing on the acute management of CVS.

Conclusion CVS is an intractable illness with a major impact on patient's quality of life. There is a long duration between symptom onset and diagnosis of the condition. There is a high association with headaches/migraines and anxiety/depression. Symptoms are more severe in adult-onset CVS. Tricyclic antidepressants have the most evidence and have high efficacy at reducing the frequency/ duration or intensity of attacks. There is limited evidence on the acute management of CVS.

\section{Abstract PWE-054 Table 1}

\begin{tabular}{llc}
\hline & $\begin{array}{l}\text { Adult-onset } \\
\text { CVS (n=446) }\end{array}$ & $\begin{array}{l}\text { Paediatric-onset } \\
\text { CVS (n=695) }\end{array}$ \\
\hline Age (mean years) & 34.0 & 8.3 \\
Age of onset (mean years) & 25.4 & 5.2 \\
Prevalence of headaches/migraines (\%) & 56.0 & 40.5 \\
Family history of headache/migraine (\%) & 56.0 & 27.8 \\
Co-existent anxiety/depression & 39.7 & 26.7 \\
Duration of CVS episode (mean days) & 5.9 & 3.4 \\
Frequency of CVS (episodes/month) & 1.2 & 0.8 \\
\hline
\end{tabular}

Competing interests None declared.

\section{PWE-055 THE PREVALENCE, CLINICAL RELEVANCE AND IMPACT ON DIET OF LACTOSE INTOLERANCE IN A POPULATION WITH LACTASE DEFICIENCY: A RANDOMISED, DOUBLE- BLIND, DOSE RESPONSE STUDY IN HEALTHY SUBJECTS AND PATIENTS WITH DIARRHOEA PREDOMINANT IRRITABLE BOWEL SYNDROME}

doi:10.1136/gutjnl-2012-302514d.55

\begin{abstract}
${ }^{1,2} \mathrm{M}$ R Fox, ${ }^{*}{ }^{3} \mathrm{~J}$ Yang, ${ }^{3} \mathrm{Y}$ Deng, ${ }^{3} \mathrm{Y}$ Cong, ${ }^{2} \mathrm{M}$ Fried, ${ }^{3} \mathrm{~N}$ Dai. ${ }^{1} \mathrm{NIHR}$ Biomedical Research Unit, Nottingham Digestive Diseases Centre, Nottingham, UK; ${ }^{2}$ Division of Gastroenterology, University Hospital Zurich, Zurich, Switzerland; ${ }^{3}$ Department of Gastroenterology, Sir Run Run Shaw Hospital, School of Medicine, Zhejiang University, Hangzhou, China
\end{abstract}

Introduction Lactose Intolerance (LI) is a common clinical syndrome; however there is a lack of high quality evidence about its epidemiology, diagnosis and clinical relevance in health and patients with functional gastrointestinal disease. Additionally the impact of LI on intake of dairy produce is uncertain because previous studies have often tested tolerance only at very high doses and/or failed to assess both self perceived and objective lactose tolerance.

Aim To assess the prevalence of LI in a population with lactose malabsorption and the effect of LI on intake of dairy products in healthy volunteers $(\mathrm{HVs})$ and patients with diarrhoea predominant irritable bowel syndrome (D-IBS).
Methods A Chinese population known to have a high prevalence of lactase deficiency was studied. $60 \mathrm{D}$-IBS patients and $60 \mathrm{HVs}$ underwent hydrogen breath test (HBT) at $10 \mathrm{~g}, 20 \mathrm{~g}, 40 \mathrm{~g}$ lactose on three test days in a randomised, double-blind three way cross-over study. Lactose Malabsorption (LM; H2 rise $>20 \mathrm{ppm}$ ) and intolerance ( $\mathrm{LI} \geq 2$ point rise on validated symptom score) were assessed at each dose. Genetic sequencing of the lactase gene promoter region was also performed. The impact of LI (both self-reported and from HBT) on lactose intake was assessed by dietary questionnaire.

Results LM was prevalent in HVs and D-IBS patients (93\% vs $92 \%$ at $40 \mathrm{~g}$ lactose, $\mathrm{p}=0.73)$. LI prevalence was lower in HVs than D-IBS patients at $10 \mathrm{~g}(3 \%$ vs $18 \%$, OR 6.51 (CI 1.38 to 30.8$), \mathrm{p}=0.008)$, $20 \mathrm{~g}(22 \%$ vs $47 \%$, OR 3.16 (CI 1.43 to 7.02$), p=0.004)$ and $40 \mathrm{~g}$ ( $68 \%$ vs $85 \%$, OR 2.63 (CI 1.08 to 6.42 ), $p=0.03$ ). The genotype in all participants was C/C-13910 and no other SNP was identified on gene sequencing of the lactase gene regulatory sequence. Most participants $(83 / 120(69 \%))$ included milk and dairy products in their diet; however D-IBS patients reported less frequent intake of dairy products than HVs and a smaller amount of lactose in the diet (D-IBS: $9.0 \mathrm{~g}(4.5-17.3)$ vs HVs: $19.5 \mathrm{~g}(6.0-36.4) ; \mathrm{p}=0.040)$. D-IBS patients also self-reported LI more frequently than $\mathrm{HVs}(63 \%$ vs $22 \%$, OR 6.25 (CI 2.78 to 14.0), p<0.001); however, self-reports of $\mathrm{LI}$ did not predict results of objective HBT.

Conclusion The likelihood of LI is increased in patients with D-IBS, especially at low lactose doses. Self reported lactose intolerance, but not objective LI on HBT, was associated with avoidance of dairy products.

Competing interests M Fox Grant/Research Support from: Nestle International, J Yang: None declared, Y Deng: None declared, Y Cong: None declared, M Fried: None declared, N Dai: None declared.

\section{PWE-056 PROSPECTIVE EVALUATION OF 403 PATIENTS WITH DIARRHOEA PREDOMINANT IRRITABLE BOWEL SYNDROME (D-IBS) FULFILLING ROME II CRITERIA}

doi:10.1136/gutjnl-2012-302514d.56

S Lin, M Kurien,* J S Leeds, D S Sanders. Department of Gastroenterology, Royal Hallamshire Hospital, Sheffield, UK

Introduction Irritable bowel syndrome (IBS) has a high prevalence with established diagnostic criteria (ROME) used to aid diagnosis. Despite increasing drives to make a positive diagnosis of IBS, patients may still require some investigations to exclude other organic conditions that may present with IBS type symptoms. There has been limited work evaluating diagnostic outcomes in this group of patients. We therefore investigated unselected patients presenting with D-IBS symptoms fulfilling ROME II criteria.

Methods Data were prospectively collected from consecutive patients meeting Rome II criteria for D-IBS in a university hospital. Demographic data, subsequent investigations and diagnostic yields of these tests were collected. All patients underwent haematologic, biochemical and immunologic testing prior to subsequent

Abstract PWE-056 Table 1 Final diagnoses in those fulfilling Rome II criteria for D-IBS

\begin{tabular}{lc}
\hline Diagnosis (total $\mathbf{n = 4 0 3 , ~ l o s t ~ t o ~ f o l l o w ~ f o l l o w - u p ~} \mathbf{n = 1 4 )}$ & Patients (\%) \\
\hline Irritable bowel syndrome & $301(75)$ \\
Pancreatic insufficiency & $28(7)$ \\
Coeliac disease & $23(5)$ \\
Diverticular disease (endoscopic/radiological finding) & $19(5)$ \\
Lactose intolerance & $8(2)$ \\
Inflammatory bowel disease & $4(1)$ \\
Small bowel bacterial overgrowth & $3(0.7)$ \\
Carcinoid & $2(0.5)$ \\
Bile acid malabsorption & $1(0.2)$ \\
\hline
\end{tabular}

